\title{
Spectrum of Imaging Manifestations and Expected Post-Treatment Findings in Thyroid Orbitopathy
}

\author{
Israel Saramago MD, Carlos Zamora MD PhD, Mauricio Castillo MD \\ University of North Carolina at Chapel Hill, Chapel Hill, NC
}

PURPOSE: Thyroid orbitopathy (TO) is the most common extrathyroidal manifestation of thyroid disease. We highlight the range of typical \& uncommon presentations as well as expected post-treatment appearance.

APPROACH/METHODS: Patients from our teaching files diagnosed with TO.

\section{FINDINGS/DISCUSSION:}

- Patients can be hyper $(90 \%)$, hypo $(5 \%)$, or euthyroid $(5 \%)$.

\section{Pathophysiology}

- Uncertain mechanism involves activation of autoantibodies against TSH receptors in extraocular muscles (EOM) \& orbital soft tissues.

- Overexpression of TSH receptors $\rightarrow$ activation of orbital fibroblasts $\rightarrow$ release of inflammatory cytokines \& differentiation into adipocytes $\rightarrow$ fatty infiltration.

- Initial inflammatory phase $\rightarrow$ progressive fibrosis $\rightarrow$ static phase.

\section{Typical findings}

- Painless exophthalmos (i.e. proptosis).

- Bilateral symmetric EOM enlargement sparing tendinous insertions in predictable fashion (I'M SLO mnemonic - beginning with inferior rectus)

- $E O M>5 \mathrm{~mm}$ generally abnormal but range is wide: medial, 3.3-5.0 mm; lateral, 1.7-4.8 mm; inferior, 3.2-6.5 mm; and superior group, 3.2-6.1 mm.

- Fatty infiltration of extraocular muscles \& retrobulbar lipomatosis

- Elevated ADC in EOM can be early finding on MRI.

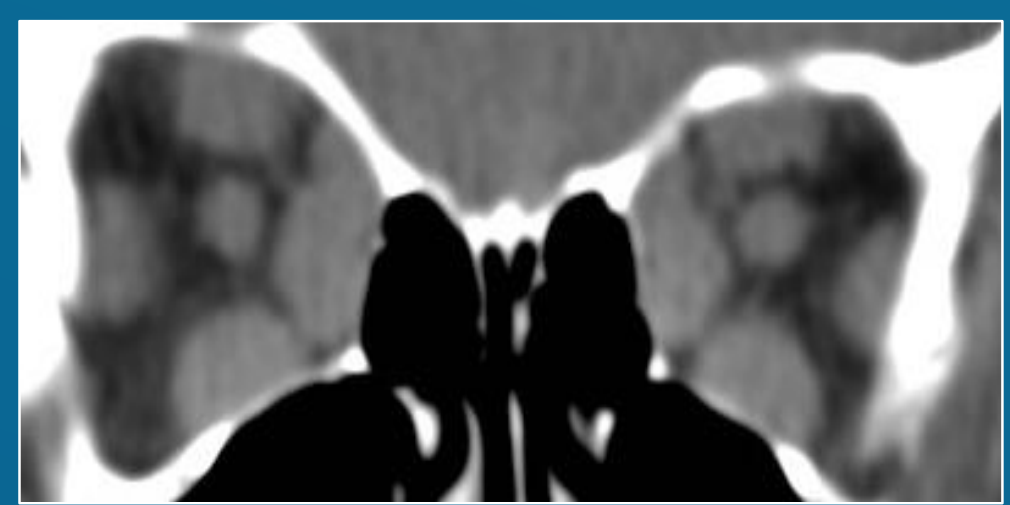

Coronal CT shows typical bilateral EOM enlargement ("I'M SLO").

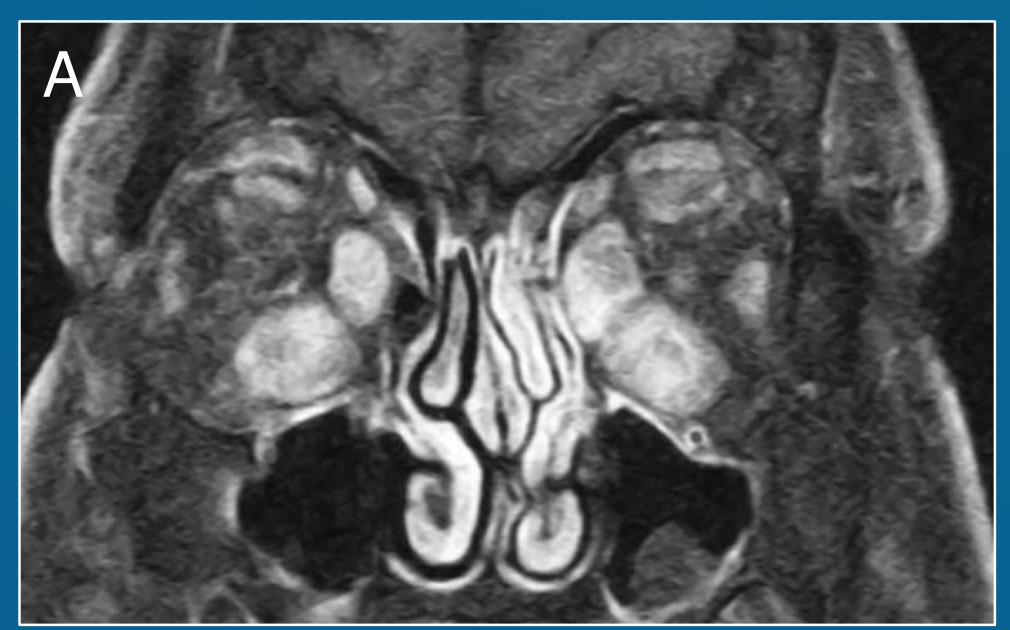

Coronal fat-saturated postcontrast T1 in 2 different patients shows extensive enhancement of EOM (A) \& intraconal fat stranding $(B)$ during the active inflammatory phase.

\section{Uncommon presentations:}

- Unilateral (10\%)

- Complicated TO in 3-5\%: compressive optic neuropathy due to orbital apex crowding $\rightarrow$ sight loss due to ischemia leading to nerve atrophy; exposure corneal ulceration due to lid retraction.

- Lacrimal gland involvement- rare, may present with worsening dry eyes.

References

1. Weiler DL. Thyroid eye disease: a review. Clin Exp Optom. 2017;100(1):20-25. doi:10.1111/cxo.12472 2. Ozgen A, Ariyurek M. Normative measurements of orbital structures using CT. AJR Am J Roentgeno. 1998;170(4):1093-1096. doi:10.2214/ajr.170.4.9530066

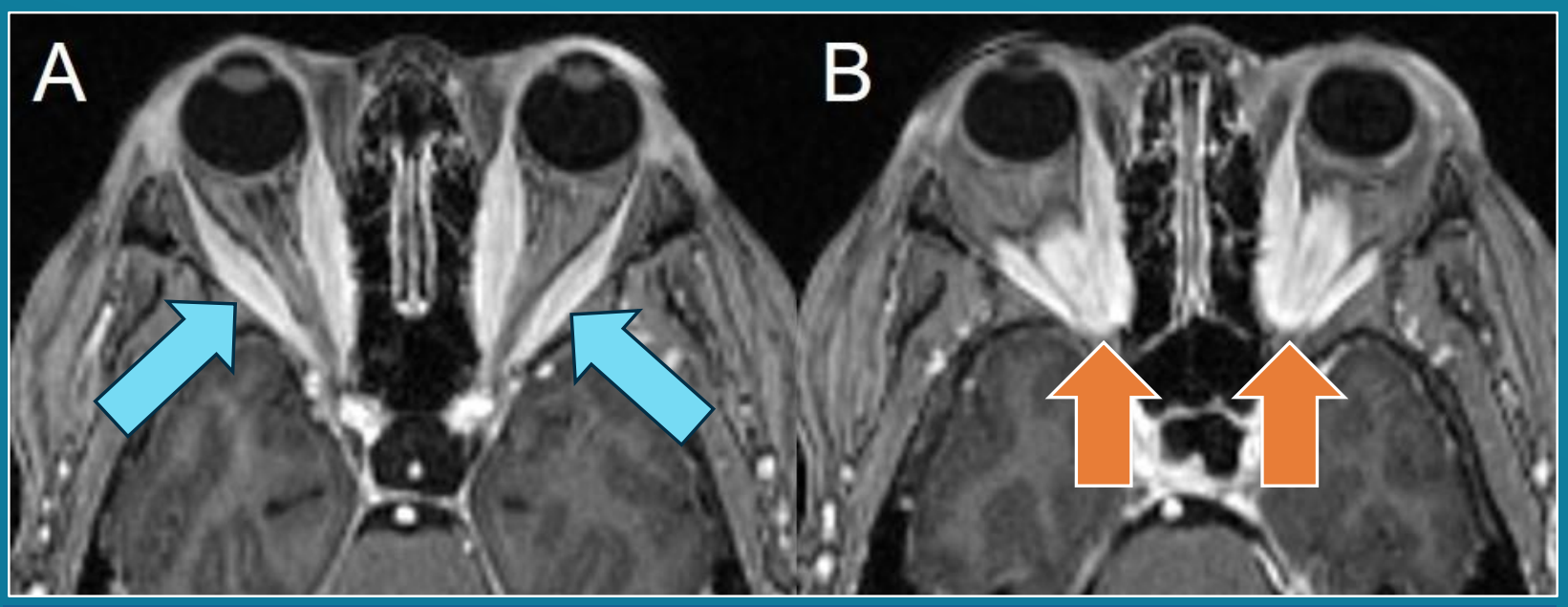

Postcontrast T1s show bilateral proptosis, enlarged EOM with diffuse enhancement (blue arrows) \& orbital apex crowding (orange arrows). Note sparing of tendinous insertions.

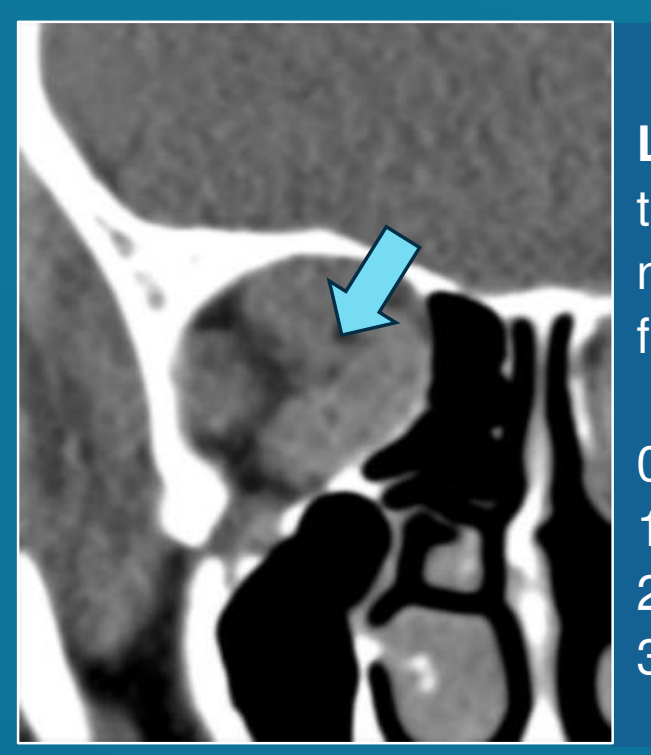

Left: effacement of perineural fat (arrow) is the most sensitive CT predictor of optic neuropathy, assessed by the Nugent score for crowding:

No perineural effacement

(mild) $1-25 \%$

(moderate) $26-50 \%$

(severe) $>50 \%$

Right: CT shows orbital fat prolapsing through bilateral superior orbital fissures (arrows) - this finding has shown variable predictive value for optic neuropathy.
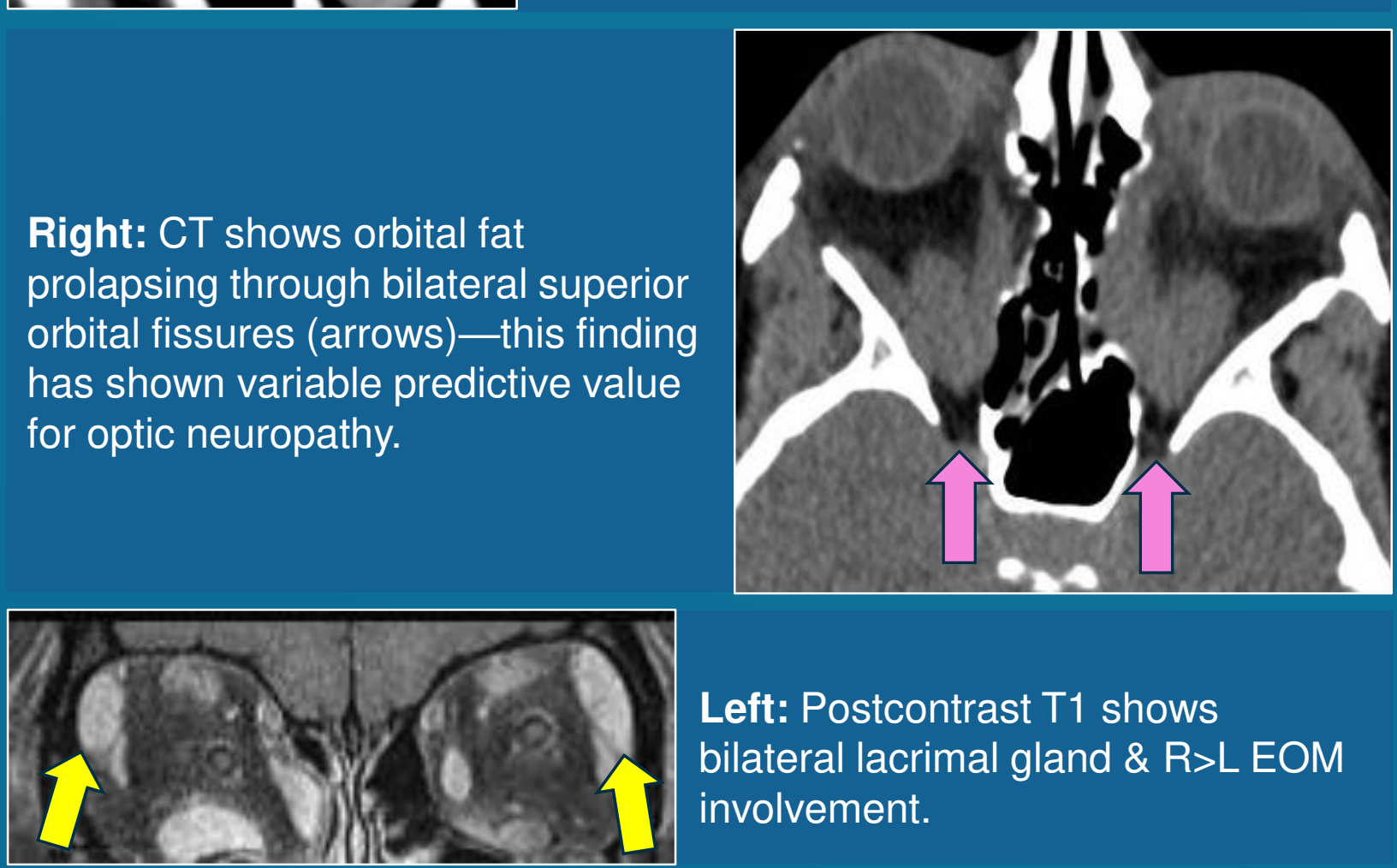

\section{Treatment}

- High dose steroids: mainstay for moderate \& severe TO

- Surgery: orbital decompression, correction of strabismus \& eyelid retraction; usually performed during static phase.

- Orbitotomy: displacement of fat and EOM towards \& beyond site of decompression.

- Radiation: Controversial, may help control active disease.

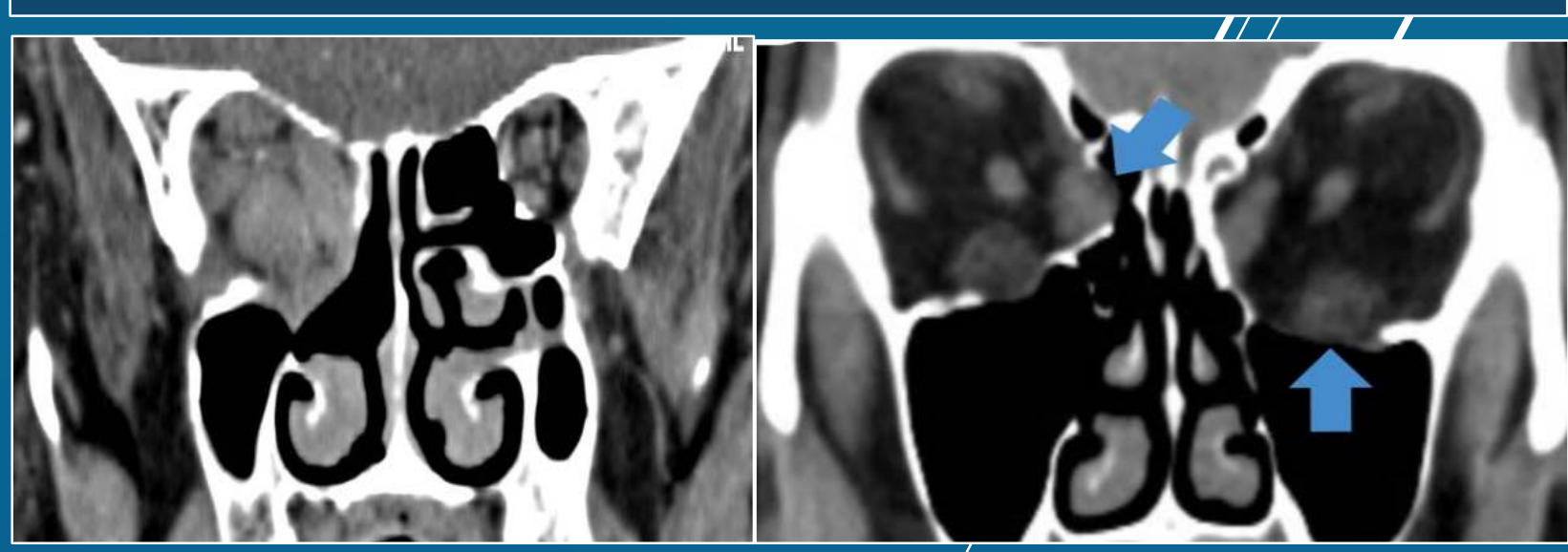

Severe unilateral right EOM orbital decompression enlargement with inferomedial
Bilateral decompression (arrows) with fatty infiltration of inferior rectus muscles.
SUMMARY/CONCLUSION: Clinical presentation \& imaging appearance of TO are variable. Patients with moderate \& severe orbital crowding are at greatest risk for optic neuropathy \& may benefit from steroids \& surgical decompression particularly in cases of sight-threatening disease. 